\title{
Active full-shell grazing-incidence optics
}

\author{
Jacqueline Roche \\ NASA Marshall Space Flight Center \\ Astrophysics Group \\ $\mathrm{X}$-ray Astronomy
}




\section{Benefits of Active Optics}

- Optical deformable mirrors

- Surface normal

- Surface parallel

- Multichannel

- Potential scientific studies

- Formation and growth of black hole seeds

- Emergence of first galaxy groups

- Details of galaxies and galaxy clusters

- X-ray astronomy desired technology

- Wide field of view

- Large effective area x-ray telescope

- High angular resolution

- MSFC focusing on full-shell grazingincidence optics

- Lightweight, tightly nested

- Manufacture, assemble and align updates

- X-ray surveyor

- Active full-shell grazing-incidence optics

- Correct Low-order figure

- Manufacturing error

- Mounting error

- Change prescription 


\section{Active Optics for Prescription Change}

- Ability to adjust optic in orbit

- optimize angular resolution for wide field of view

- optimize angular resolution for narrow field of view

- Demonstrate of ability to change optic between Wolter I and Wolter Schwarzchild

- Potential to change to polynomial prescription
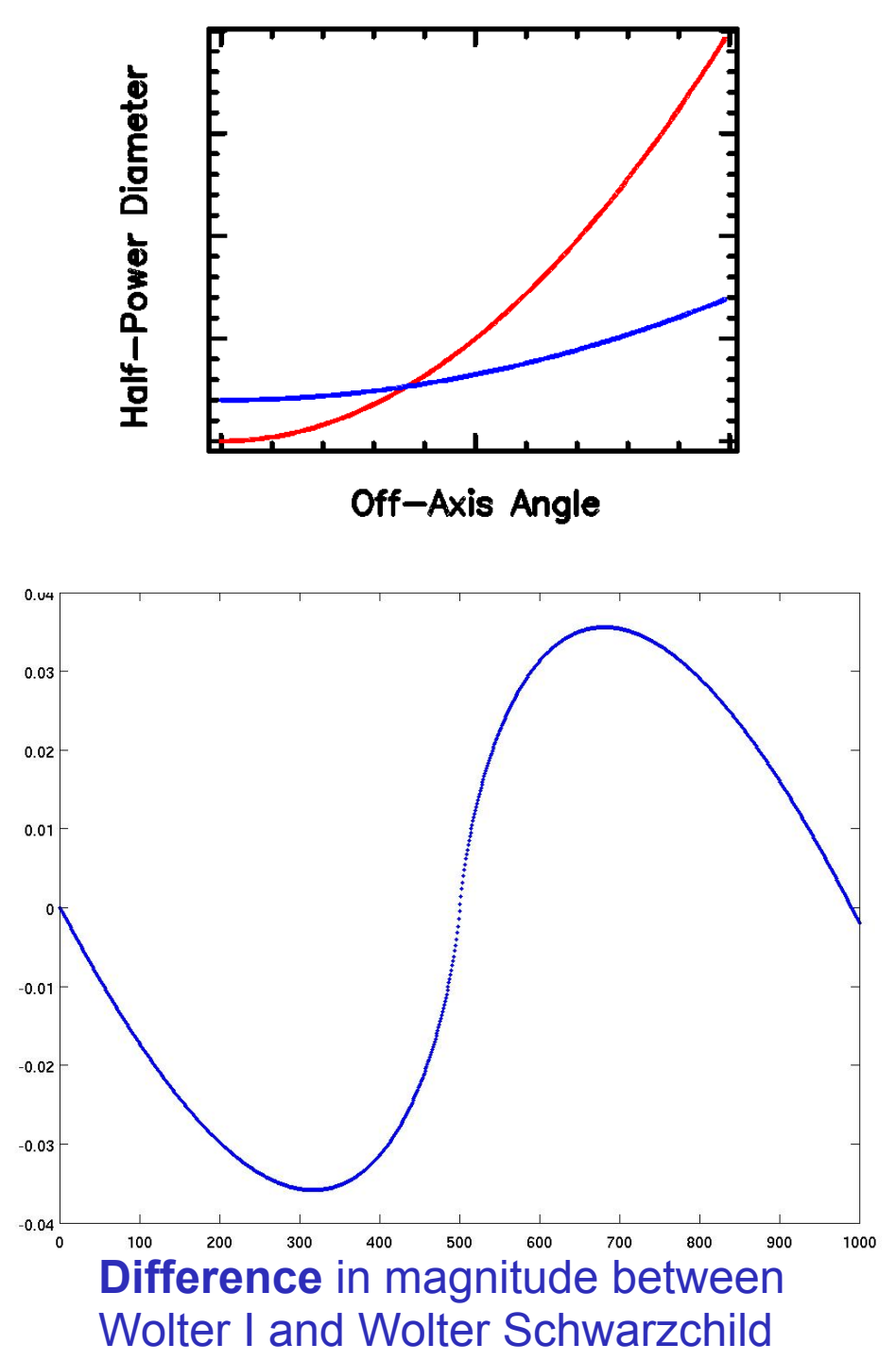
- Surface Parallel Array (SPA)

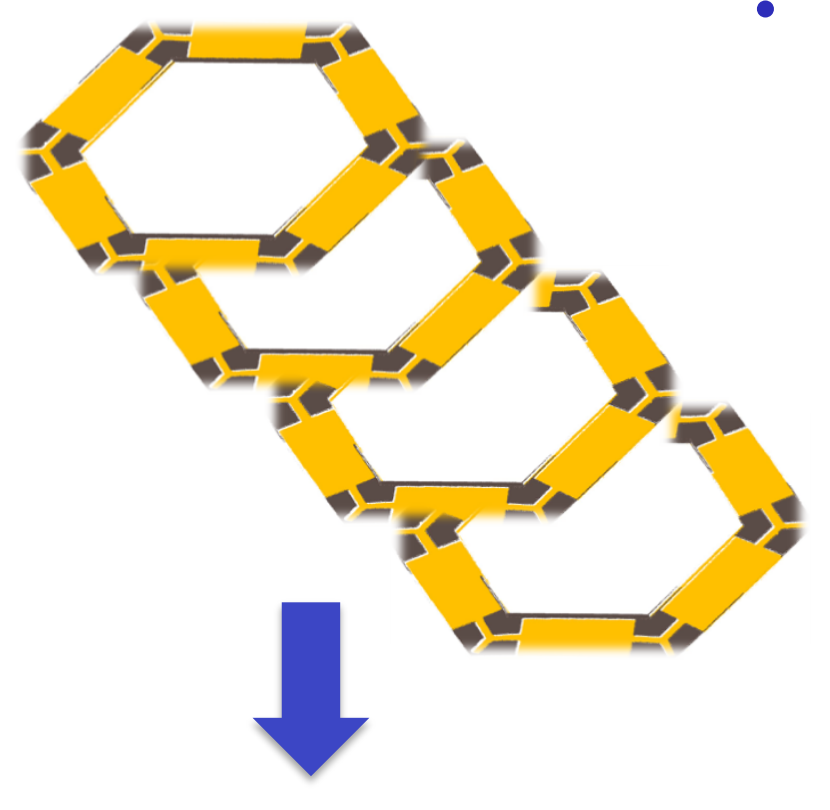

- Northrop Grumman Xinetics AOA patented technology

- Individually activated

- Bonded to thin facesheet

- Micro-machined electrostrictive ceramic actuator array

- Electrical connections are made via a flexcircuit

- Bonding performed after coating facesheet, allowing for stress balancing coating designs.

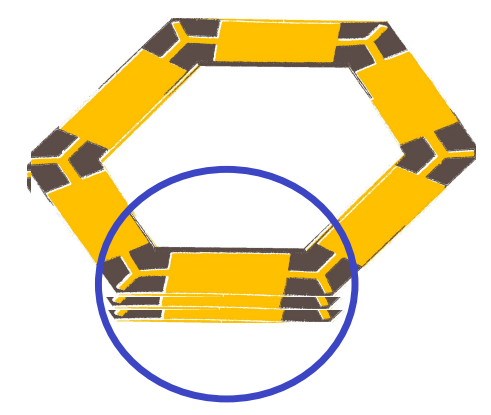

Individually activated
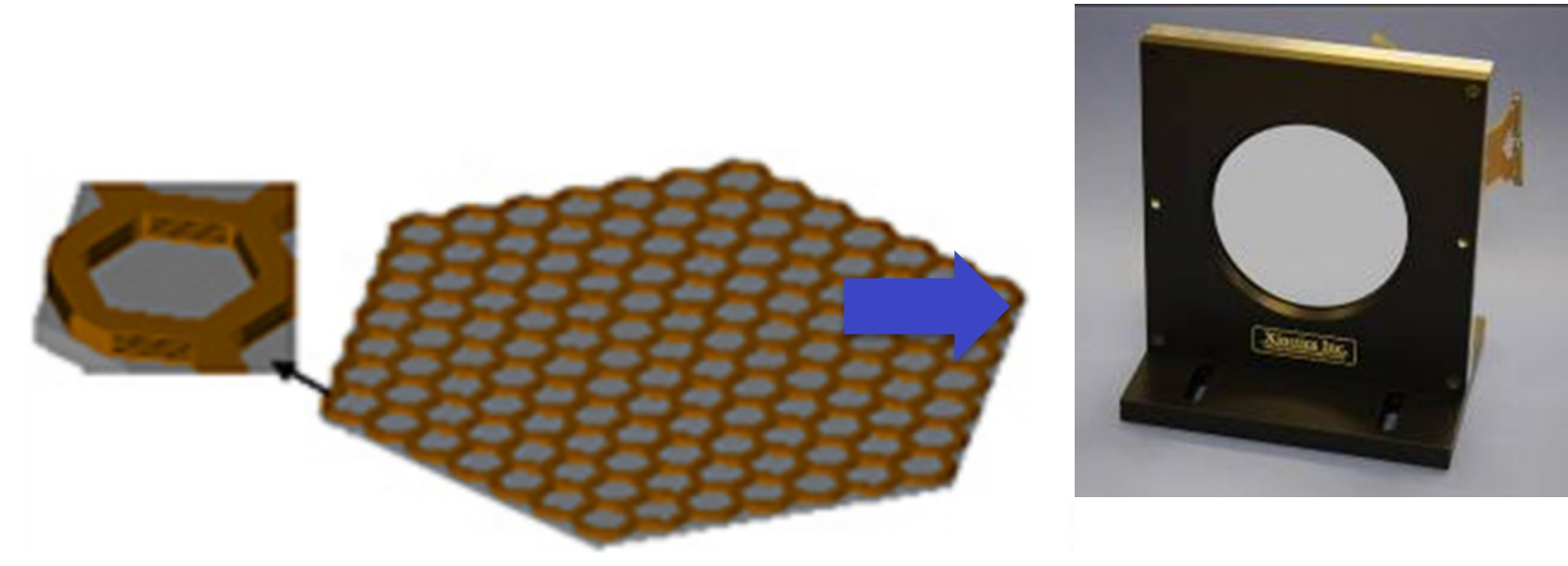


\section{Active Full-Shell Grazing-Incidence Optics}

- Bands of actuators at six positions along shell

- Using Surface Parallel Array technology

- Machine electrostrictive ceramic actuator array into bands around full-shell grazing incidence optics

- Activated together or individually

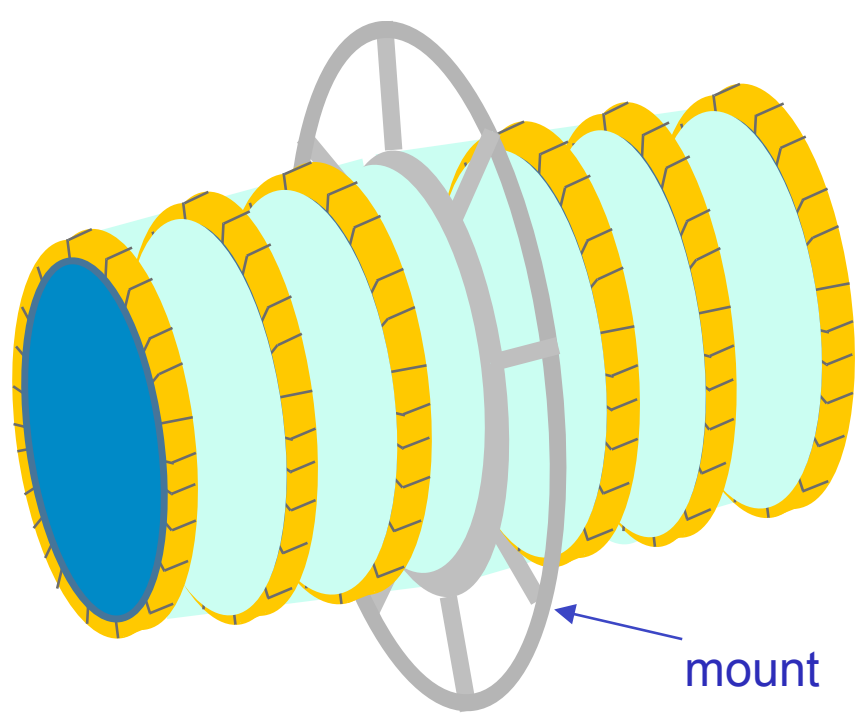

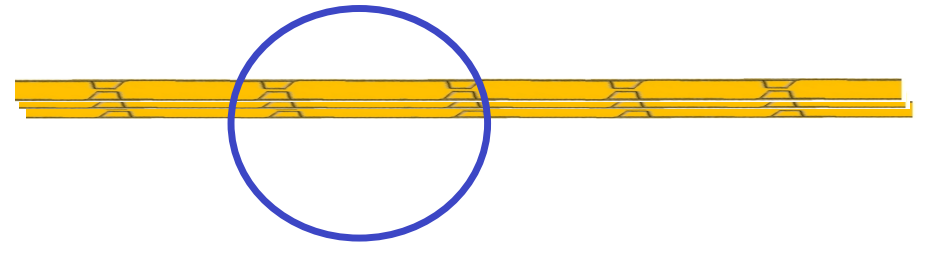

Individually activated

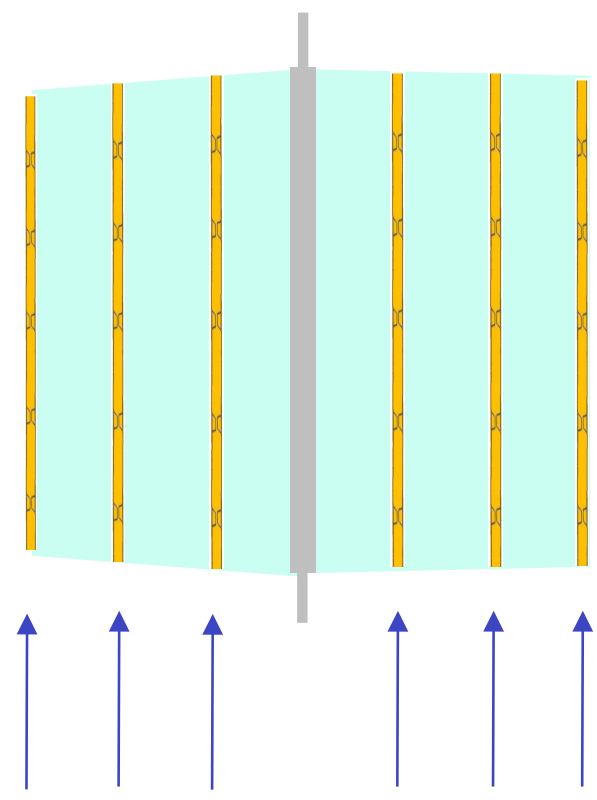

Surface Parallel Array bands 


\section{Finite Element Analysis Model}
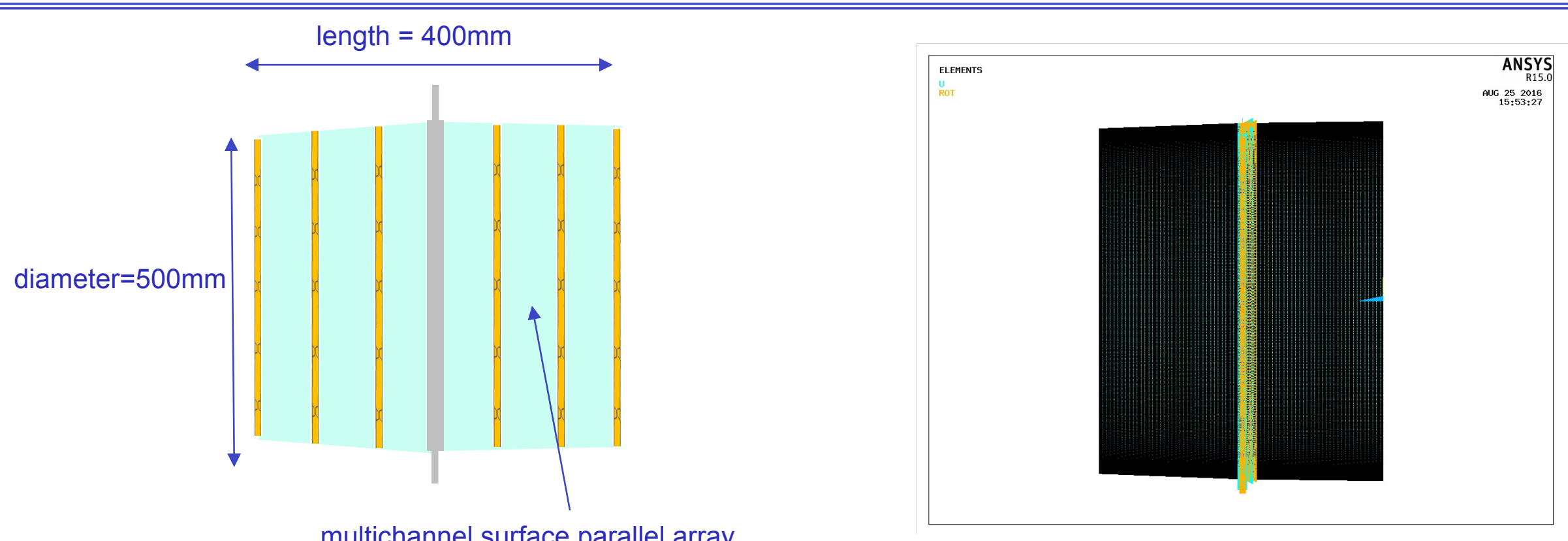

multichannel surface parallel array

- Wolter I specifications

- focal length $=10 \mathrm{~m}$

- diameter $=500 \mathrm{~mm}$

- length $=400 \mathrm{~mm}$

- thickness $=2 \mathrm{~mm}$
- Multichannel surface parallel array

- stacked PMN

- electrostrictive

- individually addressable

- Beryllium Aluminum 
Influence Functions

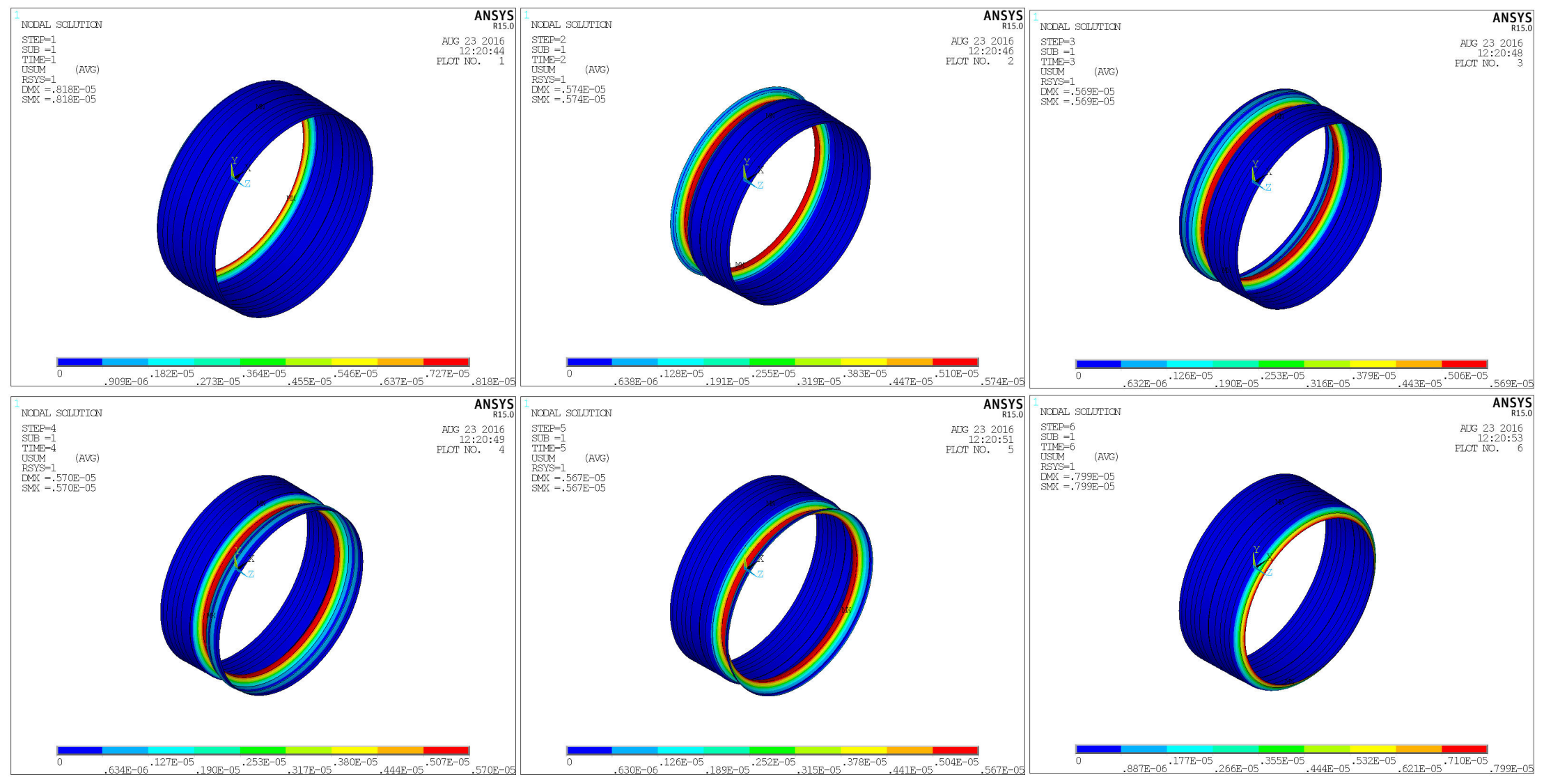




\section{Wolter Schwarzchild Adjustment}

Desired change in optical surface
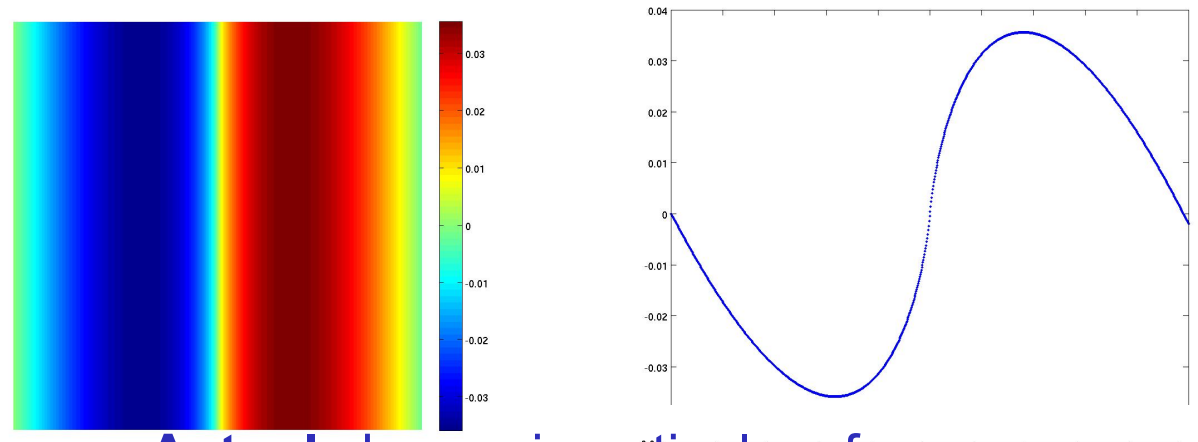

Actual change in optical surface
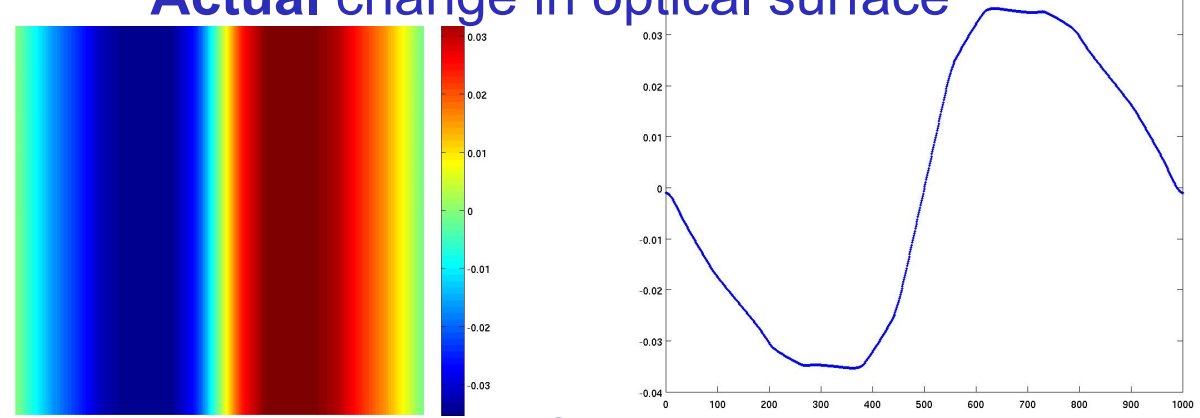

Residual figure error
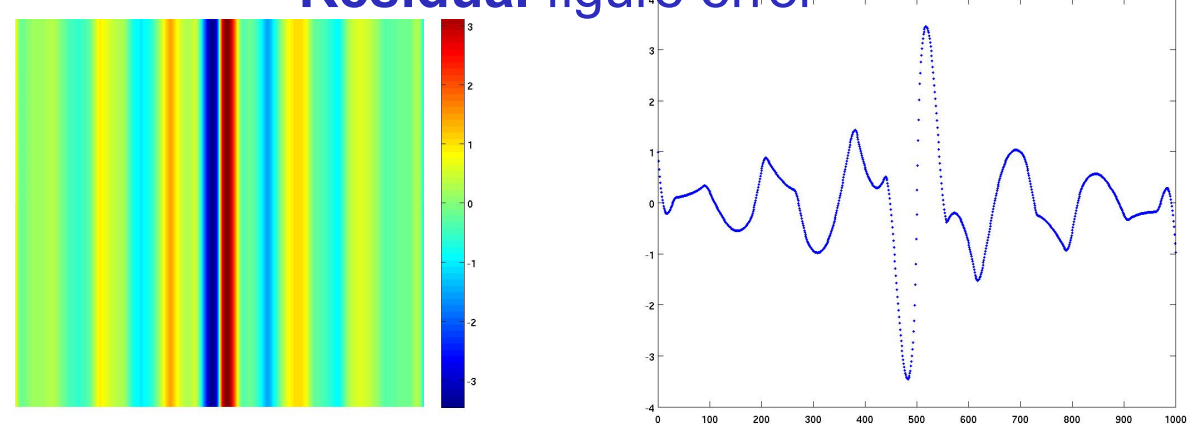


\section{Manufacturing and Mounting Correction}

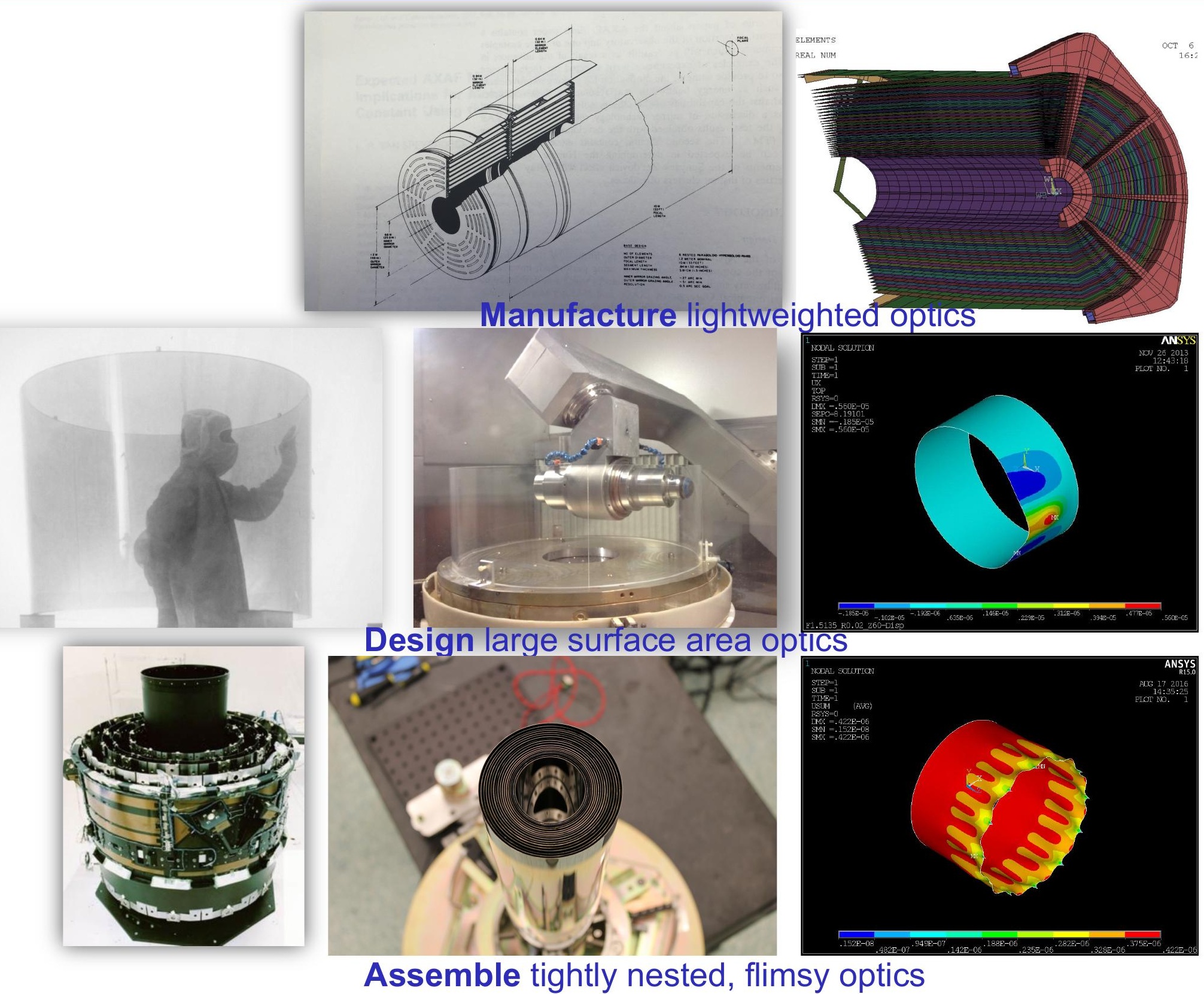




\section{Manufacturing Error Correction}
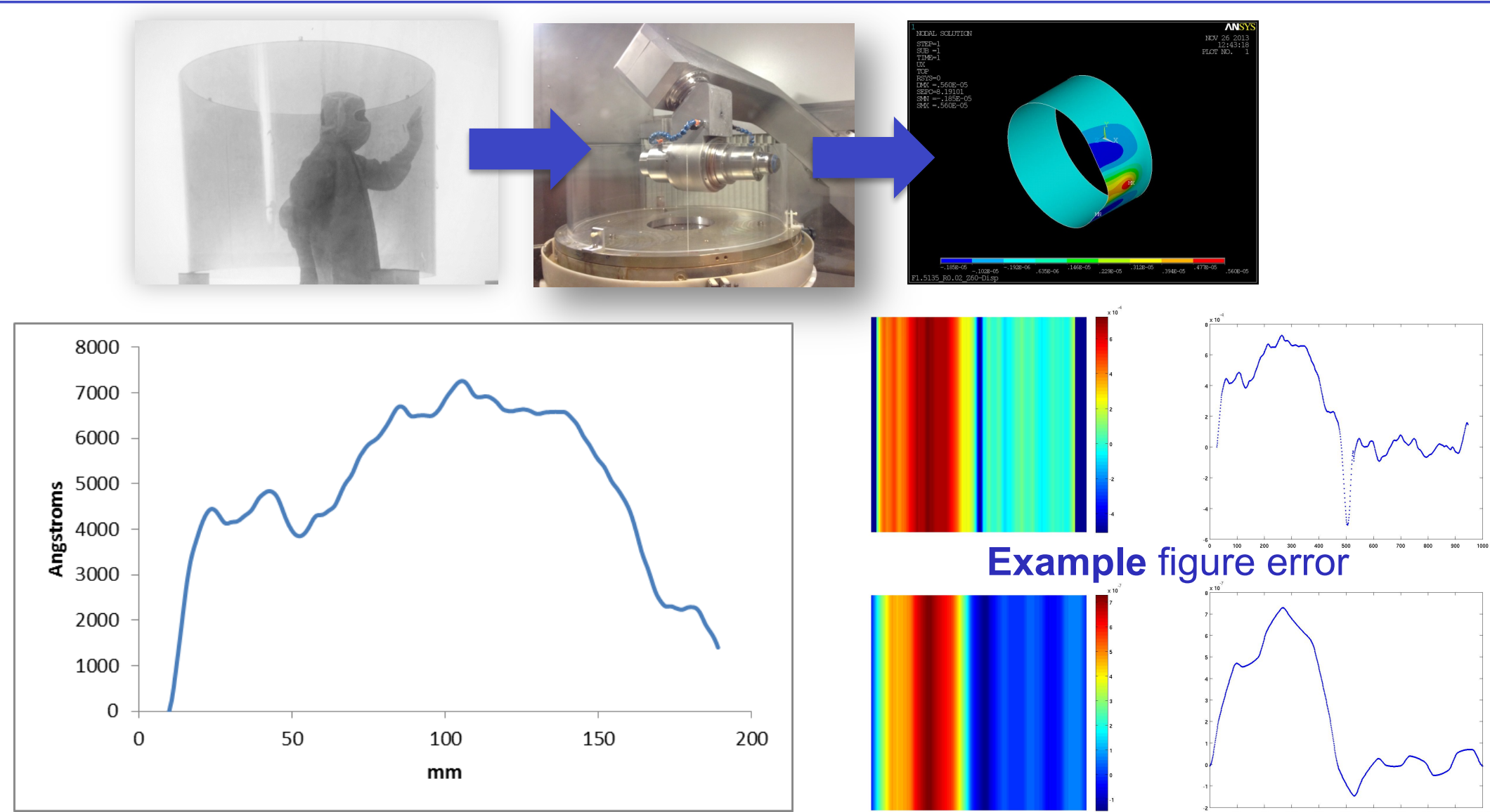

Example figure "erroror
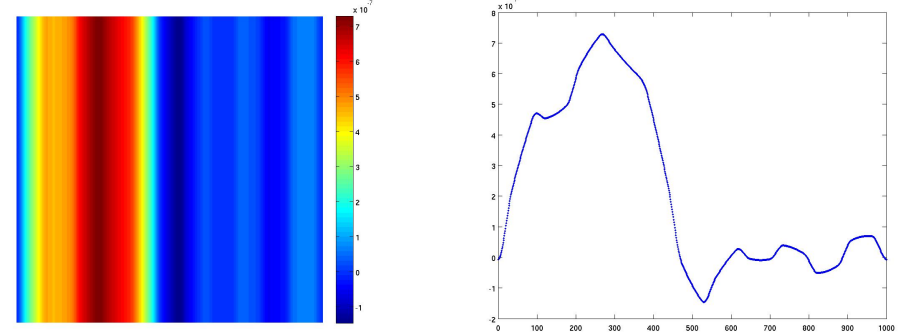

Low-order figure error correction
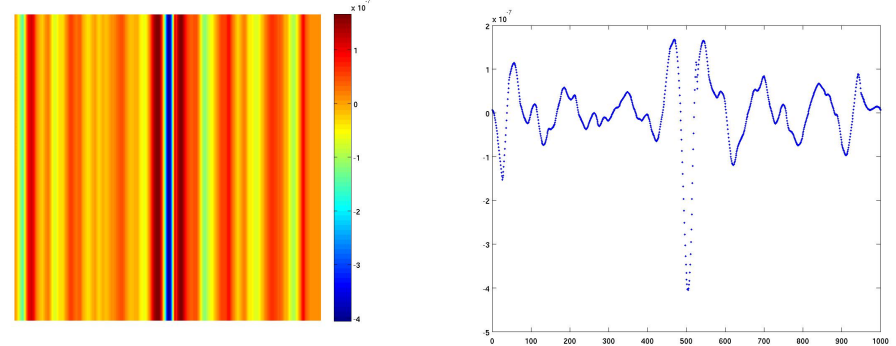

Residual figure error 


\section{Mounting Correction}

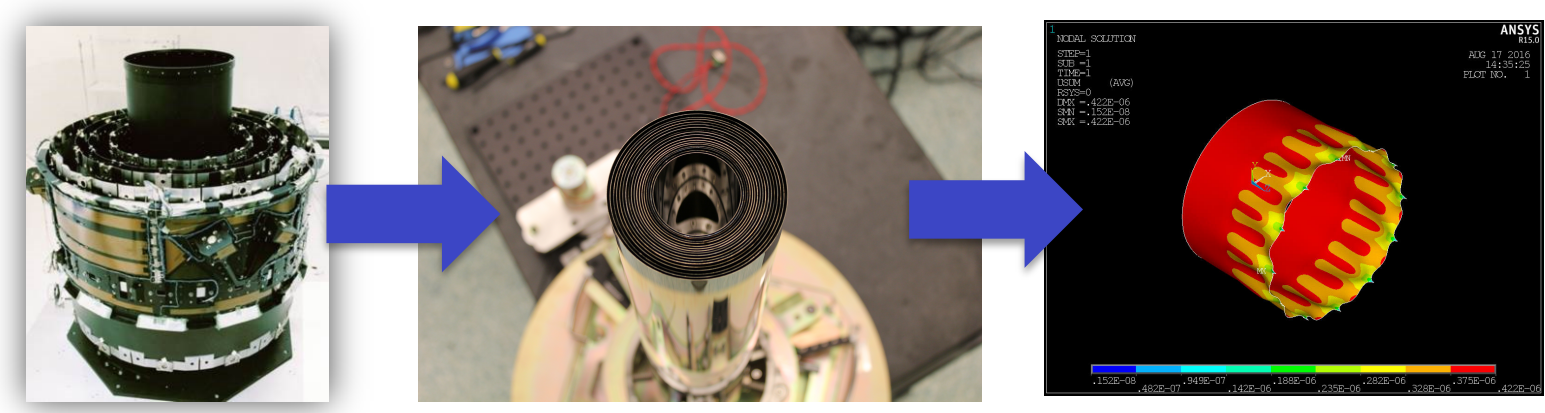

Assemble tightly nested, flimsy optics

Mounting error correction 
- Ability to adjust optic in orbit

- Correct low-order frequency figure error

- Benefits

- Small number of actuators

- Can be used in conjunction with corrective polishing and differential deposition for the high frequency errors

- Moving forward

- Polynomial

- Circumferencial correction 